\title{
Practical Solution for Effective Whole-Body Magnetic Fluid Hyperthermia Treatment
}

\author{
Hiroaki Mamiya, ${ }^{1}$ Yoshihiko Takeda, ${ }^{1,2}$ Takashi Naka, ${ }^{1}$ Naoki Kawazoe, ${ }^{1}$ \\ Guoping Chen, ${ }^{1,2}$ and Balachandran Jeyadevan ${ }^{3}$ \\ ${ }^{1}$ National Institute for Materials Science, Sengen 1-2-1, Tsukuba 305-0047, Japan \\ ${ }^{2}$ University of Tsukuba, 1-1-1 Tennodai, Tsukuba, Ibaraki 305-8577, Japan \\ ${ }^{3}$ The University of Shiga Prefecture, Hikone 522-8533, Japan \\ Correspondence should be addressed to Hiroaki Mamiya; mamiya.hiroaki@nims.go.jp
}

Received 21 June 2017; Accepted 20 November 2017; Published 13 December 2017

Academic Editor: Mohammad Mansoob Khan

Copyright (C) 2017 Hiroaki Mamiya et al. This is an open access article distributed under the Creative Commons Attribution License, which permits unrestricted use, distribution, and reproduction in any medium, provided the original work is properly cited.

\begin{abstract}
Magnetic fluid hyperthermia therapy is considered as a promising treatment for cancers including unidentifiable metastatic cancers that are scattered across the whole body. However, a recent study on heat transfer simulated on a human body model showed a serious side effect: occurrences of hot spots in normal tissues due to eddy current loss induced by variation in the irradiated magnetic field. The indicated allowable upper limit of field amplitude $H_{\mathrm{ac}}$ for constant irradiation over the entire human body corresponded to approximately $100 \mathrm{Oe}$ at a frequency $f$ of $25 \mathrm{kHz}$. The limit corresponds to the value $H_{\mathrm{ac}} f$ of $2.5 \times 10^{6}$ Oe. $\mathrm{s}^{-1}$ and is significantly lower than the conventionally accepted criteria of $6 \times 10^{7} \mathrm{Oe} \cdot \mathrm{s}^{-1}$. The present study involved evaluating maximum performance of conventional magnetic fluid hyperthermia cancer therapy below the afore-mentioned limit, and this was followed by discussing alternative methods not bound by standard frameworks by considering steady heat flow from equilibrium responses of stable nanoparticles. Consequently, the clarified potentials of quasi-stable core-shell nanoparticles, dynamic alignment of easy axes, and short pulse irradiation indicate that the whole-body magnetic fluid hyperthermia treatment is still a possible candidate for future cancer therapy.
\end{abstract}

\section{Introduction}

In principle, "if a steel pan made for induction heating (IH) cooking appliances is diced up into ultra-small pieces (magnetic nanoparticles) and selectively delivered to metastasized tiny cancers, and if the entire human body is then placed on a cooking top, all cancers including unidentifiable ones will be burned without long-term side effects, resulting in a complete cure." The realization of this scenario corresponds to an ideal therapy, and thus complete body magnetic fluid hyperthermia cancer therapy is intensively investigated by previous studies as an option in new cancer treatments to replace surgery, radiation therapy, and chemotherapy [1-8]. However, the selective annihilation of cancer cells, leaving the normal cells safe, is debatable since normal tissues are also warmed up due to eddy current loss.
The International Commission on Non-Ionizing Radiation Protection has established guidelines that are intended to protect the human body from harmful health effects by restricting exposure to electromagnetic waves [9]. The basic limit for occupational exposure to the entire body in terms of specific absorption rate (which is equal to the amount of heat generated) corresponds to $0.4 \mathrm{~mW} / \mathrm{cm}^{3}$ based on the guidelines [9]. A safety limit based on some clinical tolerance tests on healthy volunteers has been known as Brezovich criterion where $H_{\mathrm{ac}} f$ product should not go beyond $6 \times 10^{6} \mathrm{Oe} \cdot \mathrm{s}^{-1}[10]$. However, this value applies to healthy individuals, and it may not be applicable to individuals who face serious health issues. Therefore, Hergt et al. [11] proposed a maximum of approximately $H_{\text {ac }} f$ of $6 \times 10^{7}$ Oe $\cdot \mathrm{s}^{-1}$ that can be permitted for localized hyperthermia treatments of patients with serious health conditions. This value is referenced by numerous studies 
and is considered as a de facto standard for magnetic fluid hyperthermia cancer therapy. Thus, magnetic nanoparticles corresponding to an ultrasmall IH cooking pan were developed based on this irradiation condition.

Recently, Dössel and Bohnert [12] simulated the electromagnetic field distribution and the flow of heat from irradiation on a detailed human body model. The results at $f=$ $100 \mathrm{kHz}$ and $H_{\mathrm{ac}}=100$ Oe indicated that hot spots appeared in certain portions of muscles and fat in which the temperature elevation reached $10^{\circ} \mathrm{C}$ after $5 \mathrm{~min}$ despite considering the cooling effects of blood flow. The temperature elevation curves were used to estimate the thermal relaxation time $\tau$ of the hot spots that corresponded to several tens of minutes. This indicated that thermal insulation could be the reason for the existence of the hot spots. The study surmised that the allowable upper limit of $H_{\text {ac }}$ for constant irradiation over the entire human body corresponded to $100 \mathrm{Oe}$ at $f$ of $25 \mathrm{kHz}$. The limit corresponds to the value $H_{\mathrm{ac}} f$ of $2.5 \times 10^{6} \mathrm{Oe} \cdot \mathrm{s}^{-1}$. It should be noted that the condition estimated in the practical simulation significantly deviates from the long-accepted criteria. This finding is important because we cannot measure the deep body temperature during the treatment, although the improvement in accuracy for the simulation is an issue for future research.

In the present study, the new limit is considered to clarify whether or not the heating power of the present magnetic nanoparticles is sufficient to annihilate cancer cells including unidentifiable metastatic tiny cancerous areas scattered throughout the body via conventional magnetic fluid hyperthermia therapy. This is followed by a discussion on alternative strategies that are not bound by conventional frameworks of magnetic fluid hyperthermia cancer treatment. It should be noted that, with the exception of $W$, Gaussian-cgs units are used in the relationship with respect to the tissue size.

\section{Model}

2.1. Heating Power of Magnetic Nanoparticles. Magnetic hysteresis and eddy current losses heat a pan during IH cooking. Additionally, an ultrasmall pan (magnetic nanoparticles) can rotate in the applied magnetic field unlike a regularly used pan due to its small size. These rotations generate frictional heat [13]. Thus, the study begins by reexamining each heating mechanism briefly. First, with respect to magnetic hysteresis losses, the amount of heat generated, $P_{h}$, for a unit volume of magnetic nanoparticles per unit time is derived as follows:

$$
P_{h}=4 \pi \cdot f \int_{\text {Loop }} \mathbf{H} d \mathbf{M},
$$

for irradiation of the ac magnetic field, $H=H_{\mathrm{ac}} \sin (2 \pi f t)$, where $\mathbf{H}$ denotes the magnetic field and $\mathbf{M}$ denotes the magnetization. The value of the integral corresponds to the area of the hysteresis loop, and it is maximized at $4 M_{s} H_{\mathrm{ac}}$ in the case where the magnetization reversal occurs coherently in all nanoparticles when $H$ corresponds to $\pm H_{\mathrm{ac}}$, and $M_{s}$ denotes the saturation magnetization. In other words, $P_{h}$, is largest when the magnetic hysteresis loop is a square with width of
$2 H_{\text {ac }}$. However, it should be noted that it is not possible to realize such ideal reversals in actual systems. Hence, the ratio, $\eta_{\text {sq }}$, of $\int_{\text {Loop }} \mathbf{H} d \mathbf{M}$ to $4 M_{s} H_{\text {ac }}$ is considered as an index that represents the squareness of the magnetic hysteresis loop and the equation is reexpressed as follows:

$$
P_{h}=16 \pi M_{s} \eta_{\mathrm{sq}} H_{\mathrm{ac}} f .
$$

At this stage, it is necessary to consider the relaxation loss of magnetic nanoparticles that is generated when the magnetic response is given by $M=\chi^{\prime} H_{\mathrm{ac}} \sin (2 \pi f t)-\chi^{\prime \prime} H_{\mathrm{ac}} \cos (2 \pi f t)$, where $\chi^{\prime}$ and $\chi^{\prime \prime}$ denote the in-phase and out-of-phase components, respectively, of the ac susceptibility. This loss can be expressed as $4 \pi^{2} \chi^{\prime \prime} H_{\mathrm{ac}}^{2} f$ by substituting $M$ into (1). It should be noted that the relaxation loss is actually a type of hysteresis loss and $\eta_{\mathrm{sq}}=\pi \chi^{\prime \prime} H_{\mathrm{ac}} / 4 M_{s}$ is always less than unity.

Friction heat generated from the rotation of particles is considered. The work $\Delta W_{T}$ performed by the friction torque $\mathrm{T}$ while a small rotation of $\Delta \boldsymbol{\theta}$ occurs corresponds to $\mathbf{T} \cdot \Delta \boldsymbol{\theta}$. For the purpose of simplicity, it is assumed that the magnetization vector $\boldsymbol{\mu}$, which is equal to $\mathbf{M} V$, is fixed in the direction of the easy axis of particles in a manner similar to a small permanent magnet, where $V$ denotes volume of the particle. In this case, the friction dominates the rotation with respect to the inertia-less limit, and $\mathbf{T}$ always balances the magnetic torque corresponding to $4 \pi \boldsymbol{\mu} \times \mathbf{H}$. Thus, the following expression is obtained:

$$
\begin{aligned}
\Delta W_{T} & =-4 \pi \mu H \sin \theta \Delta \theta=4 \pi H \Delta(\mu \cos \theta) \\
& =4 \pi H V \Delta M_{z},
\end{aligned}
$$

where $\theta$ denotes the angle between $\mathbf{H}$ and $\boldsymbol{\mu}$ (which is in the direction of the easy axis). The amount of friction heat $P_{f}$ generated per unit volume per unit time is therefore expressed as follows:

$$
P_{f}=f \cdot \int_{\text {Loop }} \frac{d W_{T}}{V}=4 \pi f \int_{\text {Loop }} \mathbf{H} d \mathbf{M} .
$$

This equation is equivalent to (1) and indicates that the amount of energy dissipated by friction is equal to the input magnetic energy. Thus, only the names that describe the evolved heat vary depending on the focus of attention, that is, hysteresis loss or friction heat as described above.

Conversely, an eddy current loss $P_{e}$ exists that corresponds to a heat source derived from electromagnetic induction. The induced electromotive force in any closed circuit is equal to the negative of the time rate of change of the magnetic flux enclosed by the circuit, and thus the per unit volume and unit time average of $P_{e}$ for a spherical particle with electrical conductivity $\sigma$ and radius $R$ are as follows:

$$
P_{e}=\frac{4}{5} \pi^{2} \sigma R^{2} \int\left[2 \pi f H_{\mathrm{ac}}+\left(\frac{\partial M}{\partial t}\right)\right]^{2} d t .
$$

Thus, the total amount of heat generated in magnetic nanoparticles can be expressed as $P_{h}+P_{e}$, since sources of heat in the above two cases are completely different. 
2.2. Thermal Models of Tumor and Hot Spot Portion. To discuss the effectiveness of hyperthermia treatments, it is necessary to model the heat flow generated in the magnetic nanoparticles accumulated in a tumor. To consider the tumor, it is not necessary to use finite element method despite the significant dependence of thermal conductivity $\lambda$ on the organ type (typically, $0.003-0.006 \mathrm{WK}^{-1} \mathrm{~cm}^{-1}$ [14]) because a feature of this therapy includes treatments of small unidentifiable metastatic cancers with an approximate diameter $d_{c}$ of $1 \mathrm{~cm}$, within which thermal properties are assumed homogeneous. According to Andrä et al. [15], the heat at $2 \pi \lambda \Delta T d_{c}$ is roughly dissipated by conduction when such a tumor is steadily maintained at a temperature that exceeds the temperature of the surrounding area by $\Delta T$. Conversely, the blood flow in tumor tissues transfers heat at the rate $F_{b}\left(\pi d_{c}^{3} / 6\right) c_{V b} \Delta T$, where $C_{V b}$ denotes the blood specific heat and approximately corresponds to $4 \mathrm{JK}^{-1} \mathrm{~cm}^{-3}$, while $F_{b}$ corresponds to the tumor tissue blood perfusion rate and it varies based on the type, phase, size, and temperature of the tumor. Typically, $F_{b}$ is lower than that of the corresponding normal tissue and is less than $0.01 \mathrm{~s}^{-1}$ [16]. Therefore, the heating power density required to elevate the temperature of tumor with a $d_{c}$ of $1 \mathrm{~cm}$ corresponds to $\left(12 \lambda d_{c}{ }^{-2}+F_{b} C_{b}\right) \Delta T \sim$ $0.1 \Delta T \mathrm{~W} / \mathrm{cm}^{3}$ by assuming higher values for $F_{b}$ and $\lambda$ as $0.01 \mathrm{~s}^{-1}$ and $0.006 \mathrm{WK}^{-1} \mathrm{~cm}^{-1}$, respectively. For the purposes of subsequent discussion, it is necessary to remember that the thermal relaxation time in such cancers $\tau_{c}=\left(d_{c}^{2} \cdot c_{c}\right) / 27 \lambda$ corresponds to $23 \mathrm{~s}$ [17] when specific heat, $c_{c}$, is assumed to correspond to $3.7 \mathrm{JK}^{-1} \mathrm{~cm}^{-3}$.

Now, the model of the hot spot portions proposed by Dössel and Bohnert is considered. Specifically, it is necessary to simulate the temperature distributions in a human body that is exposed to ac magnetic fields by using Bio-heat equation. However, it is possible to roughly estimate the temperature of the hot spot portion $T_{h}$ by using the following simple equation because the temperature variation for each organ, reported by Dössel and Bohnert, is actually approximated by single exponential relaxation, respectively. This is expressed as follows:

$$
C_{n} \frac{d T_{h}}{d t}=P_{n}-\frac{C_{n}}{\tau_{h}}\left(T_{h}-T_{0}\right),
$$

where $P_{n}, C_{n}$, and $\tau_{h}$ denote the nominal heat generation, the specific heat, and relaxation time at the hot spot portions, respectively, and $T_{0}$ denotes the original temperature. For example, the results of curve fitting for the temperature variations of the hot spot portion in the muscle indicate that $P_{n}$ and $\tau_{h}$, correspond to $0.034 \mathrm{~W} / \mathrm{cm}^{-3}$ and $1.2 \times 10^{3} \mathrm{~s}$, respectively, for $H_{\mathrm{ac}} f=5 \times 10^{6} \mathrm{Oe} \cdot \mathrm{s}^{-1}$. For $H_{\mathrm{ac}} f=1 \times 10^{7} \mathrm{Oe} \cdot \mathrm{s}^{-1}$, $P_{n}$ and $\tau_{h}$ correspond to $0.137 \mathrm{~W} / \mathrm{cm}^{-3}$ and $1.2 \times 10^{3} \mathrm{~s}$, respectively. $C_{n}$ is set as $3.7 \mathrm{JK}^{-1} \mathrm{~cm}^{-3}$ for the above cases. It should be noted that long relaxation times indicate thermal insulation of the hot spot portion. The fact that $P_{n}$ is almost proportional to $\left(H_{\mathrm{ac}} f\right)^{2}$ is consistent with the feature of the eddy current loss, although details of actual current circuits are unknown. This indicates the validity of the approximation, and thus the hot spot temperature can be approximately calculated by using (6) with above parameters derived from the simulated results.

\section{Results and Discussion}

3.1. Conventional Magnetic Fluid Hyperthermia. For the purposes of biosafety, magnetic nanoparticles consisting of iron were examined as various types of nanomedicine because a high amount of iron $\left(0.1 \mathrm{mg} / \mathrm{cm}^{3}\right)$ is always stored in the body. Specifically, iron oxides, such as magnetite, were actually used since metallic iron is easily oxidized in the nanoform. Therefore, the survey of the conventional method is initiated by calculating the heating power of the magnetite nanoparticles by implementing actual figures. Presently, $4 \pi M_{s}, \sigma, R$, and the density are set as $5 \mathrm{kG}, 10^{4} \mathrm{Sm}^{-1}, 10 \mathrm{~nm}$, and $5.2 \mathrm{~g} / \mathrm{cm}^{3}$, respectively. Simple analyses on the results of typical hyperthermia studies indicate that the values of $\eta_{\mathrm{sq}}$ are typically in the range of $0.2-0.3$ [18]; and thus $\eta_{\mathrm{sq}}$ is corresponding to 0.3 . With respect to the condition of irradiating magnetic field, the value of $H_{\mathrm{ac}} f=2.5 \times 10^{6}$ Oe. $\mathrm{s}^{-1}$ is selected as an upper limit as stated above. The substitution of these values in (2) indicates that $P_{h}$ in this instance corresponds to $120 \mathrm{~W} / \mathrm{cm}^{3}$ (which is $23 \mathrm{~W} / \mathrm{g}$ ) when $P_{e}$ corresponds to $1 \times 10^{-4} \mathrm{~W} / \mathrm{cm}^{3}$ on the assumption that $\boldsymbol{\mu}$ is reversed in the time scale comparable to Larmor precession $\sim 10^{-9} \mathrm{~s}$. Therefore, it is possible to ignore the eddy current loss in magnetite nanoparticles in contrast to the hysteresis loss.

Conversely, it is considered that hyperthermia treatments are conventionally performed in the range of 42 $43^{\circ} \mathrm{C}$ although an established value does not exist $[1,3]$. The calculation shown in the prior section indicates that the heating power density for increasing the temperature of tumor with a $d_{c}$ of $1 \mathrm{~cm}$ by $\Delta T=5 \mathrm{~K}$ approximately corresponds to $0.5 \mathrm{~W} / \mathrm{cm}^{3}$. The heating power density can be achieved when a large amount of magnetite with $P_{h}$ of $23 \mathrm{~W} / \mathrm{g}$ is accumulated at a concentration of $22 \mathrm{mg} / \mathrm{cm}^{3}$ in tumor tissues. In contrast, Huang and Hainfeld [19] reported that they injected ferric oxide nanoparticles intravenously via a tail vein, and, consequently, the results indicated that nanoparticles accumulated in the tumor at a concentration of approximately $1.9 \mathrm{mg} / \mathrm{cm}^{3}$. This concentration corresponds to the top class performance at present. However, it is significantly less than the required figures. Thus, the current discussion indicates that conventional hyperthermia treatment using magnetite nanoparticles is impractical for unidentifiable tiny cancers areas scattered throughout the body without significant advancements in drug delivery technology in the future.

An easy method involves eliminating the focus on the whole-body treatment by drug delivery techniques and focusing instead on identified larger cancer treatments in a local area by direct injection. This is because the heat generated by eddy current loss in hot spot portions is proportional to the cross-sectional area through which the magnetic flux passes as discussed above. Hence, restricting an irradiation range to local area suppresses temperature elevation in the hot spot portion. Furthermore, the temperature elevation of tumors can become significant as the size of tumors increases 
because the heat dissipation is proportional to the area of the cancer surface while the heating power afforded by the accumulated nanoparticles is proportional to the volume. Additionally, a direct injection on the identified cancers enables the enhancement of the concentration of nanoparticles inside tumors when compared with that in other drug delivery techniques. However, we know that larger cancerous area at known positions can be also removed through surgery. Therefore, it is still important to examine other solutions for the whole-body treatment by considering points overlooked in conventional magnetic fluid hyperthermia treatments.

3.2. Alternative Strategies. As described in the preceding section, the conventional hyperthermia treatment using magnetite nanoparticles is ineffective with respect to tiny cancers scattered throughout the body. To resolve this, it is necessary to shift the standpoint by considering steady heating by using the equilibrium response of stable nanoparticles to those for temporary heating from a nonequilibrium response of quasistable nanoparticles in order to clarify the manners to maximize each factor in (2). First, the saturation magnetization $M_{s}$ is considered. For example, only iron oxide nanoparticles were commercially used for magnetic fluids in machines due to their excellent oxidization resistance, although metallic iron has much higher $M_{s}$. This is because industrial products generally need durability exceeding ten years. On the basis of such experience, iron oxide nanoparticles have been examined for hyperthermia treatments. Nevertheless, it should be noticed that the hyperthermia treatment period is significantly shorter. Hence, the iron oxide shell, iron core nanoparticles can be considered as a candidate despite instability for long-term usage. This substance includes a large value of $M_{s}$ for the iron core and biocompatibility of the iron oxide shell. Previous studies indicated that the nanoparticles with this type of structure possess a saturation magnetization $4 \pi M_{s}$ of $12 \mathrm{kG}$ at $14 \mathrm{~nm}$ [20] and $16 \mathrm{kG}$ at $40 \mathrm{~nm}$ [21], which is twice that of iron oxide. Little is, however, known as to their long-term durability. Thus, the stability of magnetization for the commercially available coreshell nanoparticles $\left(4 \pi M_{s}=12 \mathrm{kG}\right)$ obtained from BoutiQ Nanoparticle Solutions was checked by using an extraction method (PPMS, Quantum Design). Consequently, the results indicated that the magnetization did not decline after six months from when the substance was received as shown in Figure 1. As expected, the iron oxide of the shell also serves as a surface protection layer. Hence, it is applicable for magnetic fluid hyperthermia treatments because such treatments are typically completed in half year at most, although it may become difficult to maintain excellent dispersion stability for over several months because the larger $M_{s}$ strengthens effects of the interparticle interactions.

Subsequently, the squareness of magnetic hysteresis loop, $\eta_{\mathrm{sq}}$, is considered. As is widely known, alignment of easy axes of single domain nanoparticles improves the squareness, and thus easy axes orientation in static magnetic fields are widely employed prior to sintering permanent magnets to improve their performance [22]. In contrast to these types of ceramics, the easy axes of nanoparticles, starts to thermally fluctuate immediately after cutting the static magnetic field in the

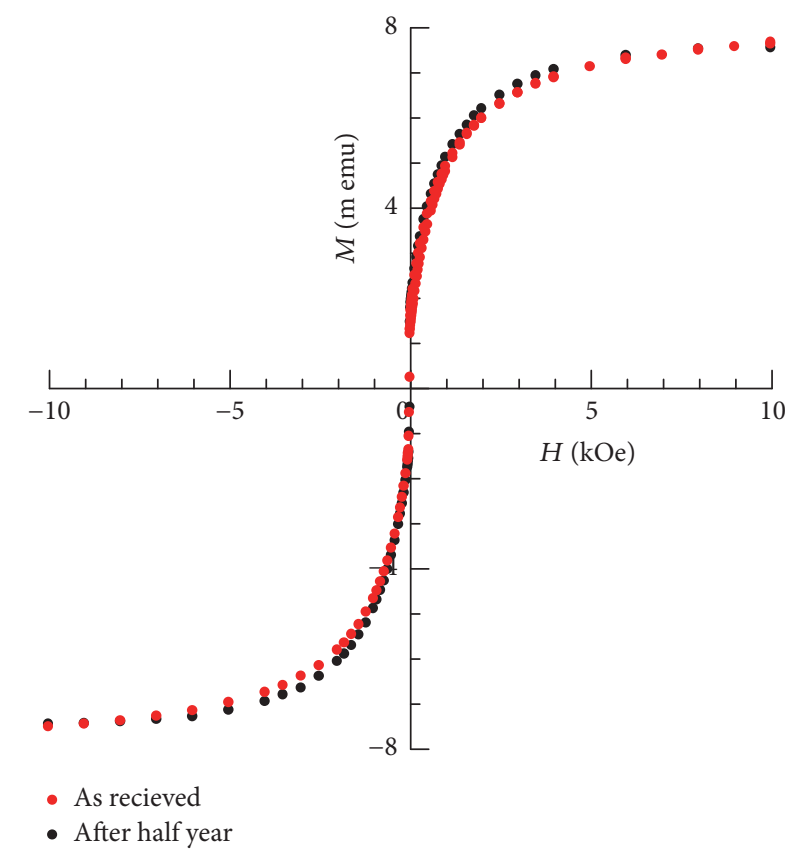

FIGURE 1: Magnetization curves of magnetic fluid containing Fe/FeO core/shell nanoparticles. The red dots indicate the curve immediately after the nanoparticles are received while the black dots show the curve after half a year has elapsed.

human body. On the other hand, the continuous application of a large static magnetic field biases the field variation range from the hysteresis loop. Therefore, it is actually difficult to increase the value by $\eta_{\mathrm{sq}}$ by using a static magnetic field. Recently, a study employed a Brownian dynamics simulation for rotatable magnetic nanoparticles and indicated that these types of nanoparticles form nonequilibrium structure in ac magnetic fields and the orientation of the easy axes occurs under a certain condition [13]. Therefore, the capability to steadily align the easy axes is examined by applying an ac magnetic field using an in situ small angle X-ray scattering experiment in static and ac magnetic fields. Insets in Figure 2 show the two-dimensional contour diagrams of the intensity of X-rays scattered by magnetite nanoparticles (a) in a zero magnetic field, (b) in static magnetic field of $1000 \mathrm{Oe}$, and (c) in an AC magnetic field with $H_{\mathrm{ac}}$ of $660 \mathrm{Oe}$ and $f$ of $100 \mathrm{kHz}$, where the average size, $M_{s}$, and the coercivity of the nanoparticles correspond to $17.4 \mathrm{~nm}, 3.5 \mathrm{kG}$, and $53 \mathrm{Oe}$, respectively. The findings readily indicate that the contour lines change from circles to diamonds when static as well as ac magnetic fields are applied. The intensity dependence of circumferential angle symmetry is fourfold not only in a static magnetic field, but also in an AC magnetic field with comparable oscillation in amplitude as shown in Figure 2. These results indicate that, apart from the details, the ac magnetic field can steadily align the cuboidal magnetite nanoparticles in a manner similar to the static magnetic field. This fact is consistent with the prediction of the previous Brownian dynamics simulation, which indicated that $\eta_{\mathrm{sq}}$ can be increased to 0.8 [13]. Consequently, the squareness of the magnetic hysteresis loop can be improved by increasing $H_{\mathrm{ac}}$ 


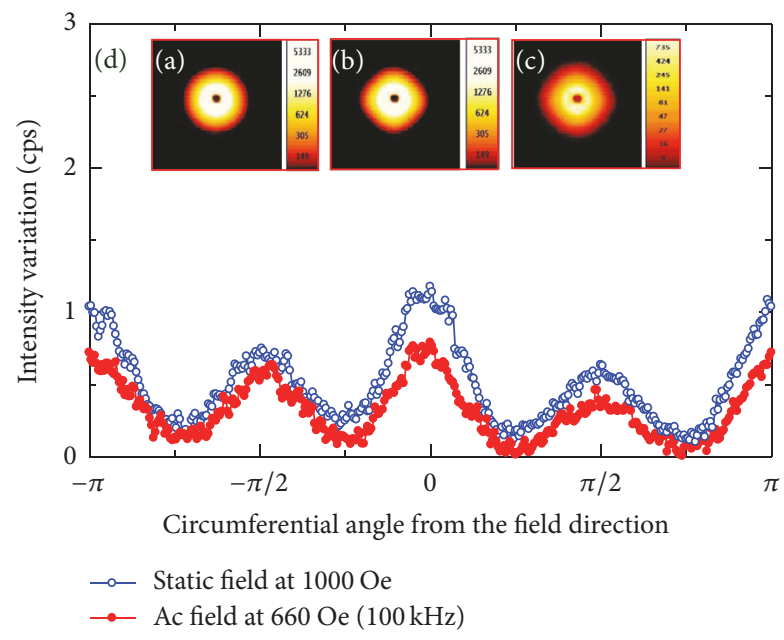

Figure 2: Two-dimensional contour diagrams of small angle Xray scattering intensity for magnetite nanoparticles (a) in a zero magnetic field, (b) in static magnetic field of 1000 Oe, and (c) in ac magnetic field with $H_{\mathrm{ac}}$ of $660 \mathrm{Oe}$ and $f$ of $100 \mathrm{kHz}$. (d) Circumferential angle dependencies of small angle X-ray scattering intensities in a scattering vector range from $0.4 \mathrm{~nm}^{-1}$ to $0.6 \mathrm{~nm}^{-1}$.

and decreasing $f$ even when the value of $\left(H_{\mathrm{ac}} f\right)^{2}$ is restricted to suppress the occurrence of hot spots.

Prior to examining the next topic, it is necessary to focus attention on the issue of the switching field distribution of magnetic nanoparticles. As widely known [22], the existence of the switching field distribution reduces the squareness of the magnetic hysteresis loop, and thus it is necessary to prepare magnetic nanoparticles without anisotropy dispersion. However, there is very little agreement with respect to the effective origin of magnetic anisotropy even for iron oxide nanoparticles that are widely used for over half a century, not to mention the recently developed core-shell nanoparticles. Hence, clarification of the origin is another critical issue.

Finally, it is necessary to consider the remaining parameters, $H_{\mathrm{ac}}$ and $f$, subject to the occurrence of hot spots. Recalling daily experiences, the main point here is that exposure to high temperatures even only for a short period of time leads to skin burns. For example, the time required for thermal damage corresponds to a couple of minutes at $50^{\circ} \mathrm{C}$ and to several hours at $44^{\circ} \mathrm{C}$ [23]. Interestingly, extant studies did not focus on this type of temporary heating by pulsed irradiation in the development of magnetic nanoparticles for hyperthermia treatments, although researchers in the field of hyperthermia treatments always considered irradiation duration. Hence, the present study does not consider the fixed idea that the treatments are performed in the steady state and considers the possibility of short pulse heating at high magnetic field amplitudes prior to storing the heat in the thermally insulated tissues. An example with $H_{\text {ac }} f$ of 1 $\times 10^{7} \mathrm{Oe} \cdot \mathrm{s}^{-1}(10 \mathrm{kHz}, 1000 \mathrm{Oe})$ is considered. With respect to this applied magnetic field, easy axes orientation with $\eta_{\mathrm{sq}}$ of 0.8 is assumed for the above-mentioned core-shell nanoparticles with $4 \pi M_{s}$, of $16 \mathrm{kG}$ considered as an ideal case. The concentration of nanoparticles in the tumor involves

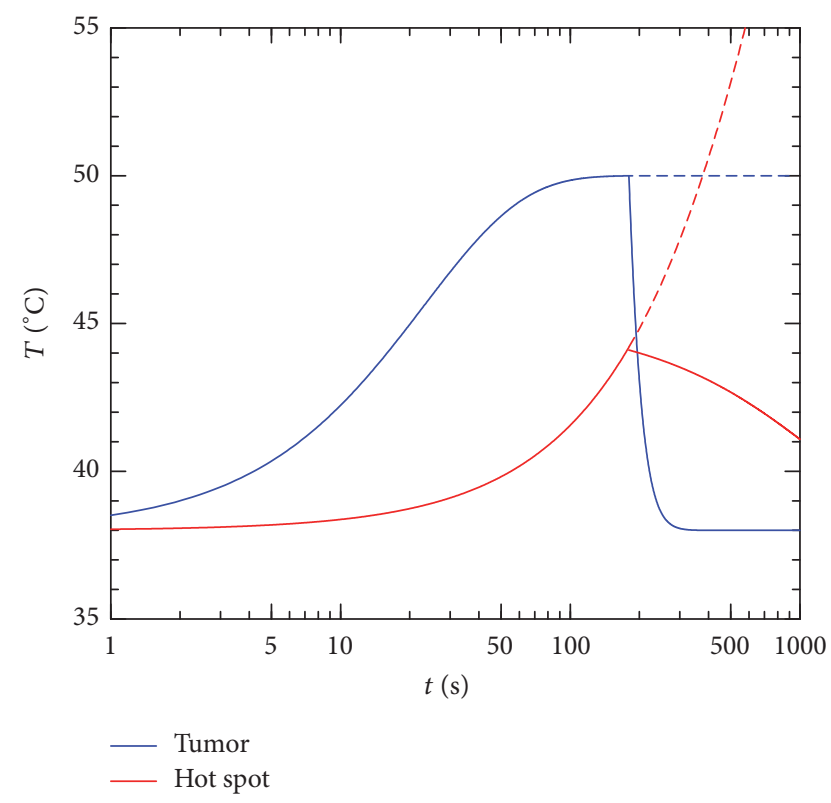

FIgURE 3: Calculated heating curves of tiny tumors and hot spot portions in normal tissues by pulsed irradiation of ac magnetic field. Details are described in the text.

setting the practical value at $2 \mathrm{mg} / \mathrm{cm}^{3}$ [19]. Under these conditions, the maximum generated heat corresponds to $1.2 \mathrm{~W} / \mathrm{cm}^{3}$ inside the tiny tumor with a $d_{c}$ of $1 \mathrm{~cm}$. Under these conditions, a simple calculation using the above-mentioned $\tau_{c}$ of $23 \mathrm{~s}$ and $c_{c}$ of $3.7 \mathrm{JK}^{-1} \mathrm{~cm}^{-3}$ indicates that the temperature of tiny tumors would exceed $49^{\circ} \mathrm{C}$ for an exposure time corresponding to $t=60 \mathrm{~s}$ as shown in Figure 3. Then, when the irradiation ceases at a time $t$ of $180 \mathrm{~s}$, the temperature drops rapidly. In this case, the tumor is burned at $50^{\circ} \mathrm{C}$ for $2 \mathrm{~min}$, which is sufficient to damage the tumor.

It is necessary to check the temperature at the hot spot portions in normal tissues under the afore-mentioned conditions. The temperature elevation under the same condition of $H_{\mathrm{ac}} f=1 \times 10^{7}$ Oe $\cdot \mathrm{s}^{-1}$ curve was calculated by using (6) and $0.137 \mathrm{~W} / \mathrm{cm}^{-3}, 3.7 \mathrm{JK}^{-1} \mathrm{~cm}^{-3}$, and $1.2 \times 10^{3} \mathrm{~s}$ for $P_{n}, C_{n}$, and $\tau_{h}$, respectively. The result shown in Figure 3 indicates that the temperature reaches $44^{\circ} \mathrm{C}$ at $t=180 \mathrm{~s}$. If the irradiation is continued constantly, then the temperature increases to $83^{\circ} \mathrm{C}$ finally. Conversely, the irradiation is terminated immediately after the temperature reached $44^{\circ} \mathrm{C}$ in the example. Consequently, the temperature of normal tissues does not exceed $44^{\circ} \mathrm{C}$ even at the hot spot portions as indicated by the solid line. The thermal damage for a couple of minutes is negligible below $44^{\circ} \mathrm{C}$. Thus, this calculation indicates that it is possible to eradicate a metastatic cancer of roughly $1 \mathrm{~cm}$ that was not identified in the diagnosis without serious side effects on normal tissues if irradiation can be performed by using the ideal magnetic nanoparticles in this manner. The required concentration of the nanoparticles accumulated in this type of a metastatic cancer corresponds to $2 \mathrm{mg} / \mathrm{cm}^{3}$, which is one tenth of that in the conventional method and it is achievable within the framework of the current drug delivery technology. 


\section{Conclusions}

In a conventional magnetic fluid hyperthermia therapy for cancers including unidentifiable metastatic ones scattered in the whole body needs selective accumulation of the magnetic nanoparticles at a concentration of $22 \mathrm{mg} / \mathrm{cm}^{3}$, which is significantly higher than the value achieved by current drug delivery technique. Thus, the present study proposes a shift from techniques based on static, steady, and equilibrium to unstable, dynamic, and nonequilibrium scenario, although other kinds of approaches using assistance of ultrasonic or photothermal hyperthermic effects have been discussed $[24,25]$. Therefore, the possibility of temporary heating of tumors was examined by pulsed irradiation using dynamically aligned structures of quasi-stable core-shell nanoparticles. Consequently, the results indicated that the proposed manner allows ideal whole-body cancer treatment at a realistic nanoparticle concentration via current drug delivery techniques. However, we must notice that much still remains to be done in each issue. First, it is not possible to control magnetic anisotropy even in widely used magnetite nanoparticles and especially in the recently developed coreshell nanoparticles. Additionally, there is a paucity of research on the dynamic alignment of easy axes of nanoparticles and especially of interacting nanoparticles. Furthermore, it is necessary to simulate heat transfers during temporary heating by pulsed irradiation in a detailed human body model in the next stage. These issues can be examined through cross-field collaboration since they are correlated with each other. For example, significance of the synthesis of coreshell nanoparticles with higher magnetization decreases if dynamic aligned structures are not formed due to an unbalance between the anisotropy field of the synthesized particles and the amplitude $H_{\mathrm{ac}}$, which is optimized in the heat transfer simulation in a detailed human body. However, it is necessary for this simulation to input heating power of nanoparticles that depend on both $M_{s}$ and $\eta_{\mathrm{sq}}$. Although there are many technical challenges to overcome as discussed here, magnetic fluid hyperthermia therapy continues to be a promising candidate for ideal treatments of cancers including unidentifiable metastatic cancers scattered throughout the body, preventing the side effects of the occurrence of hot spots as pointed out recently.

\section{Disclosure}

The funders had no role in study design, data collection and analysis, decision to publish, or preparation of the manuscript.

\section{Conflicts of Interest}

The authors declare that they have no conflicts of interest.

\section{Authors' Contributions}

All authors conceived the study. Hiroaki Mamiya performed the experiments, analyzed the data, and wrote the paper.

\section{Acknowledgments}

This work was partly supported by Japan Society for the Promotion of Science (JSPS) KAKENHI Grants nos. 24310071 (Hiroaki Mamiya and Balachandran Jeyadevan) and 15K13278 (Hiroaki Mamiya).

\section{References}

[1] Q. A. Pankhurst, N. K. T. Thanh, S. K. Jones, and J. Dobson, "Progress in applications of magnetic nanoparticles in biomedicine," Journal of Physics D: Applied Physics, vol. 42, Article ID 224001, 2009.

[2] K. M. Krishnan, "Biomedical nanomagnetics: a spin through possibilities in imaging, diagnostics, and therapy," IEEE Transactions on Magnetics, vol. 46, no. 7, pp. 2523-2558, 2010.

[3] B. Jeyadevan, "Present status and prospects of magnetite nanoparticles-based hyperthermia," Nippon Seramikkusu Kyokai Gakujutsu Ronbunshi, vol. 118, no. 1378, pp. 391-401, 2010.

[4] H. Mamiya, "Recent advances in understanding magnetic nanoparticles in ac magnetic fields and optimal design for targeted hyperthermia," Journal of Nanomaterials, vol. 2013, Article ID 752973, 17 pages, 2013.

[5] S. Dutz and R. Hergt, "Magnetic nanoparticle heating and heat transfer on a microscale: basic principles, realities and physical limitations of hyperthermia for tumour therapy," International Journal of Hyperthermia, vol. 29, no. 8, pp. 790-800, 2013.

[6] E. A. Périgo, G. Hemery, O. Sandre et al., "Fundamentals and advances in magnetic hyperthermia," Applied Physics Reviews, vol. 2, no. 4, Article ID 041302, 2015.

[7] M. Molcan, V. Petrenko, M. Avdeev et al., "Structure characterization of the magnetosome solutions for hyperthermia study," Journal of Molecular Liquids, vol. 235, pp. 11-16, 2017.

[8] Y. Shlapa, S. Solopan, A. Bodnaruk et al., "Lanthanumstrontium manganites for magnetic nanohyperthermia: Fine tuning of parameters by substitutions in lanthanum sublattice," Journal of Alloys and Compounds, vol. 702, pp. 31-37, 2017.

[9] International Commission on Non-Ionizing Radiation Protection, "Guide-lines for limiting exposure to time-varying electric, magnetic, and electro-magnetic fields (up to $300 \mathrm{GHz}$ )," Health Phys, vol. 74, p. 494, 1998.

[10] I. A. Brezovich, "Low frequency hyperthermia: Capacitive and ferromagnetic thermoseed methods," Medical Physics Monograph, vol. 16, pp. 82-111, 1988.

[11] R. Hergt, S. Dutz, and M. Röder, "Effects of size distribution on hysteresis losses of magnetic nanoparticles for hyperthermia," Journal of Physics: Condensed Matter, vol. 20, no. 38, Article ID 385214, 2008.

[12] O. Dössel and J. Bohnert, "Safety considerations for magnetic fields of $10 \mathrm{mT}$ to $100 \mathrm{mT}$ amplitude in the frequency range of 10 $\mathrm{kHz}$ to $100 \mathrm{kHz}$ for magnetic particle imaging," Biomedizinische Technik. Biomedical Engineering, vol. 58, no. 6, pp. 611-621, 2013.

[13] H. Mamiya and B. Jeyadevan, "Hyperthermic effects of dissipative structures of magnetic nanoparticles in large alternating magnetic fields," Scientific Reports, vol. 1, article 157, 2011.

[14] C. Rossmanna and D. Haemmerich, "Review of temperature dependence of thermal properties, dielectric properties, and perfusion of biological tissues at hyperthermic and ablation temperatures," Critical Reviews in Biomedical Engineering, vol. 42, no. 6, pp. 467-492, 2014. 
[15] W. Andrä, C. G. D’Ambly, R. Hergt, I. Hilger, and W. A. Kaiser, "Temperature distribution as function of time around a small spherical heat source of local magnetic hyperthermia," Journal of Magnetism and Magnetic Materials, vol. 194, no. 1, pp. 197203, 1999.

[16] C. W. Song, "Effect of local hyperthermia on blood flow and microenvironment: a review," Cancer Research, vol. 44, pp. 4721s-4730s, 1984.

[17] R. R. Anderson and J. A. Parrish, "Selective photothermolysis: precise microsurgery by selective absorption of pulsed radiation," Science, vol. 220, no. 4596, pp. 524-527, 1983.

[18] E. Kita, T. Oda, T. Kayano et al., "Ferromagnetic nanoparticles for magnetic hyperthermia and thermoablation therapy," Journal of Physics D: Applied Physics, vol. 43, no. 47, Article ID 474011, 2010.

[19] H. S. Huang and J. F. Hainfeld, "Intravenous magnetic nanoparticle cancer hyperthermia," International Journal of Nanomedicine, vol. 8, pp. 2521-2532, 2013.

[20] D. A. J. Herman, P. Ferguson, S. Cheong et al., "Hot-injection synthesis of iron/iron oxide core/shell nanoparticles for T 2 contrast enhancement in magnetic resonance imaging," Chemical Communications, vol. 47, no. 32, pp. 9221-9223, 2011.

[21] Y. Qiang, J. Antony, A. Sharma, J. Nutting, D. Sikes, and D. Meyer, "Iron/iron oxide core-shell nanoclusters for biomedical applications," Journal of Nanoparticle Research, vol. 8, no. 3-4, pp. 489-496, 2006.

[22] T. Maki and S. Hirosawa, "Analysis of squareness in demagnetization curve of $\mathrm{Nd}-\mathrm{Fe}-\mathrm{B}$ magnet produced by the hydrogenation-disproportionation-desorption-recombination process," Journal of Applied Physics, vol. 103, no. 4, Article ID 043904, 2008.

[23] A. R. Moritz and F. C. Henriques, "Studies of thermal injury 11. The relative importance of time and surface temperature in the causation of thermal bums," American Journal of Pathology, vol. 23, pp. 695-720, 1947.

[24] A. Józefczak, K. Kaczmarek, T. Hornowski et al., "Magnetic nanoparticles for enhancing the effectiveness of ultrasonic hyperthermia," Applied Physics Letters, vol. 108, no. 26, Article ID 263701, 2016.

[25] N. Alegret, A. Criado, and M. Prato, "Recent Advances of Graphene-based Hybrids with Magnetic Nanoparticles for Biomedical Applications," Current Medicinal Chemistry, vol. 24, no. 5, pp. 529-536, 2017. 

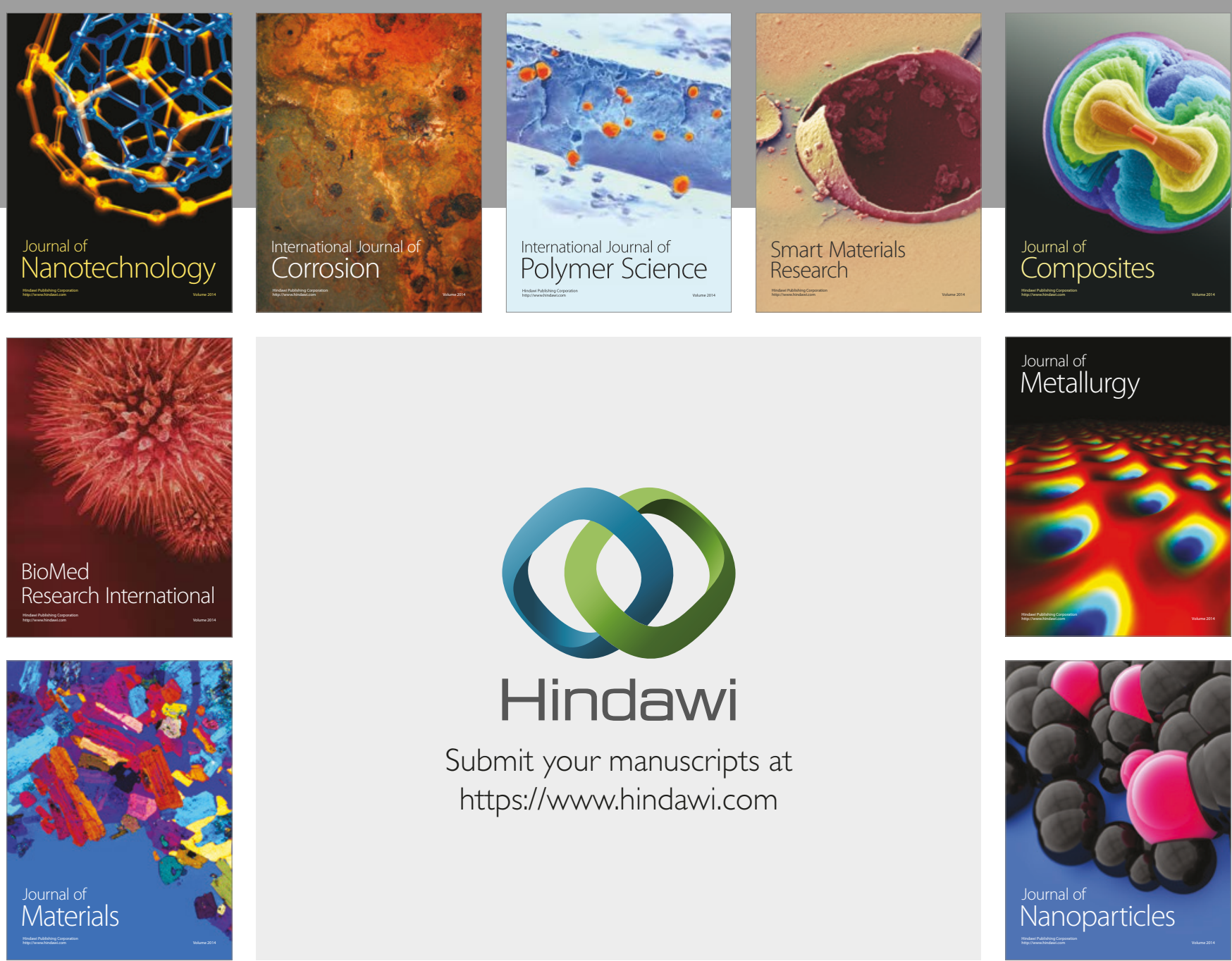

\section{Hindawi}

Submit your manuscripts at

https://www.hindawi.com
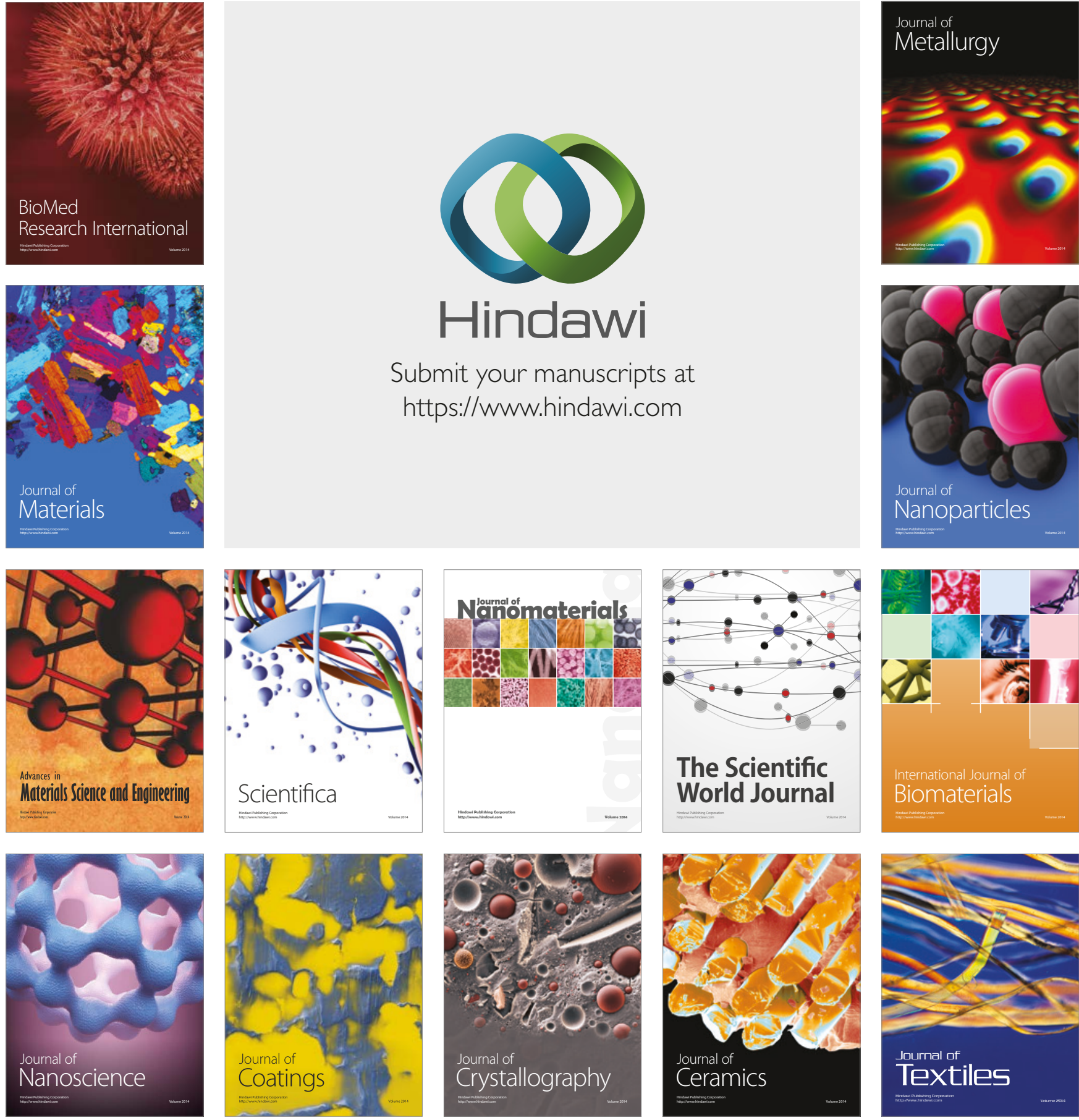

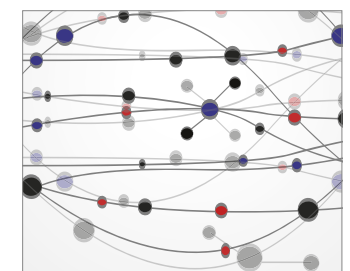

The Scientific World Journal
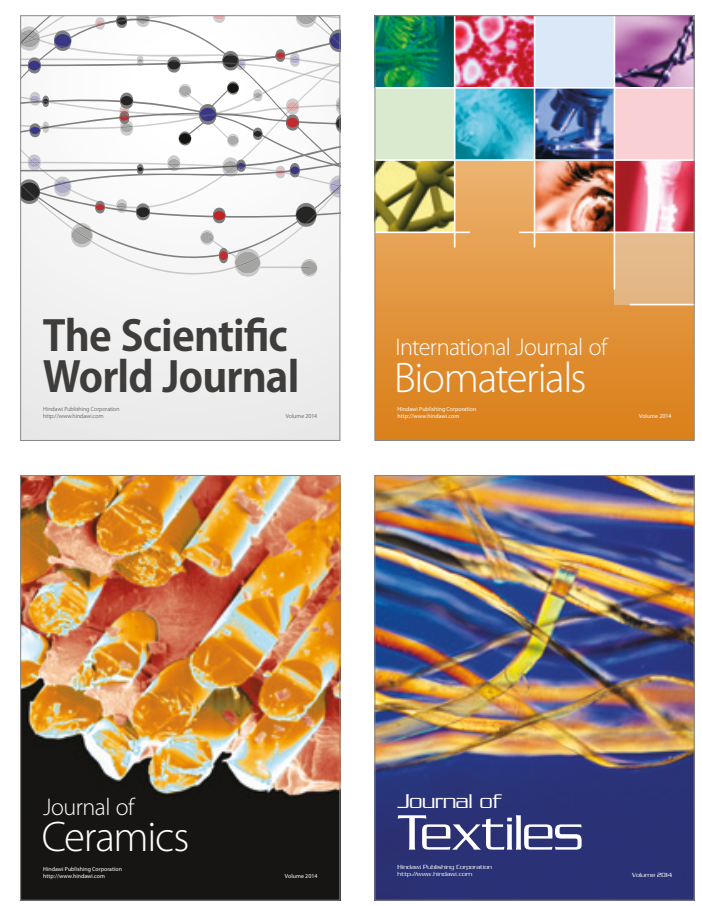